\title{
UNITÉ DU VIVANT, BIOMIMÉTISME : CONFÉRENCES D'ERIK ORSENNA ET GILLES BOEUF LORS DE LA SÉANCE SOLENNELLE DE L'ACADÉMIE VÉTÉRINAIRE DE FRANCE
}

\author{
UNITY OF THE LIVING WORLD, BIOMIMICRY: CONFERENCES BY ERIK \\ ORSENNA AND GILLES BOEUF AT THE SOLEMN SESSION OF THE \\ FRENCH VETERINARY ACADEMY
}

Par Agnès FABRE-DELOYE ${ }^{1}$ et Jean Luc ANGOT²

(Compte rendu de la séance solennelle du 3 décembre 2020)

Mots-clés : Vivant, Biomimétisme, Bio-inspiration, Académie Vétérinaire de France

Key words: Living world, biomimicry, bio-inspiration, French Veterinary Academy

Lors de sa séance solennelle du 3 décembre 2020, l'Académie vétérinaire de France a eu l'honneur d'accueillir Erik Orsenna, de l'Académie française, et Gilles Boeuf, ancien président du Muséum national d'histoire naturelle. Erik Orsenna a donné une conférence sur l'unité du Vivant et Gilles Boeuf sur le biomimétisme et la bio-inspiration.

\section{L'UNITÉ DU VIVANT}

Travailler sur les interdépendances passionne Erik Orsenna, économiste de formation. C'est pour cette raison qu'il a écrit sur la mondialisation, en commençant par le végétal : le coton et ensuite l'eau. Erik Orsenna rapporte en préambule les propos de Claude Lévi-Strauss, qui se demandait comment on pouvait être seulement économiste et donc n'avoir qu'une vision extrêmement partielle de la réalité et ceux de François Jacob, qui fut son voisin à l'Académie française pendant douze ans et qui n'a pas cessé de lui reprocher d'occuper le fauteuil $n^{\circ} 17$, celui de Louis Pasteur. Il lui disait en plus «non seulement tu es économiste mais tu es romancier, donc tu as comme morale le mensonge! ". Erik Orsenna précise ensuite «Et puis heureusement j'ai rencontré Gilles Boeuf qui est mon professeur de vie et qui m'a mis les idées en place ". Il rappelle avoir rédigé une biographie de Pasteur, ce qui lui a permis de se rendre compte de la notion d'Une Santé et de l'interdépendance absolue entre tous les échelons du vivant. "Pasteur était méprisé par les médecins qui l'appelaient le chimiatre. Or Pasteur a travaillé les $9 / 10^{i}$ de sa vie sur les animaux et c'est ce travail sur les animaux qui lui a permis de sauver des êtres humains".
Erik Orsenna évoque également son livre "Géopolitique des moustiques ". Pour parler du Vivant l'écrivain académicien, qui se définit comme un raconteur d'histoires, "un géographe manqué, un vétérinaire manqué et un médecin manqué " et non comme un essayiste ou un philosophe, a cherché un "personnage " pour son dernier ouvrage. Il l'a trouvé avec le cochon. Pourquoi cet animal ? Parce que l'auteur habite la côte nord de la Bretagne, l'un des lieux les plus importants de production de porcs en France. Ensuite, parce que ce suidé est lié à l'être humain à la fois par l'histoire, la géographie, la génétique, et enfin du fait de l'explosion de l'épidémie de peste porcine africaine. Il a donc commencé à travailler en même temps sur le cochon et sur la peste porcine. L'écrivain avoue ne pas avoir été du tout surpris par la pandémie arrivée récemment, car possédant une sorte de culture des épidémies de par les modèles animaux étudiés pour ses ouvrages. En travaillant sur les moustiques, il avait déjà constaté le lien entre l'évolution climatique et l'accroissement des risques. Selon Erik Orsenna, nous avons toujours essayé de répondre à la demande des consommateurs qui veulent en fait payer toujours moins cher leur nourriture. Il regrette que la part du budget des ménages réservée à l'alimentation soit passée en une vingtaine d'années de $30 \%$ à $11 \%$. Il aborde également le thème sensible du bien-être animal et celui de la surdensité dans les élevages. Il évoque la fabrication de "viande " non animale : «c'est quelque chose qui est fou car ce n'est pas de la viande puisque ce n'est pas animal ". Erik Orsenna s'est en outre élevé contre les atteintes à l'environnement. La conviction d'Erik Orsenna est

(1) Chargée de mission, chargée d'enseignement, École nationale vétérinaire d'Alfort, DPASP, 7 avenue du Général de Gaulle 94700 Maisons-Alfort Cedex. Courriel : agnes.fabre-deloye@vet-alfort.fr

(2) Président 2020 de l'AVF, Inspecteur général de santé publique vétérinaire, Ministère de l'agriculture et de l'alimentation, 251 , rue de Vaugirard 75732 Paris Cedex 15. Courriel : jean-luc.angot@agriculture.gouv.fr 
que la prochaine crise après la crise sanitaire sera celle de l'eau. " tous les deltas $d u$ monde sont menacés. Or 500 millions d'êtres humains habitent les deltas ". En conclusion, Erik Orsenna a indiqué être intervenu auprès du Chef de l'Etat pour plaider l'intégration d'un vétérinaire au Conseil scientifique Covid-19. Le président de l'AVF informe Erik Orsenna de sa nomination comme membre d'honneur de l'AVF et le félicite.

\section{BIOMIMÉTISME ET BIO-INSPIRATION}

Gilles Boeuf s'est intéressé au biomimétisme dès les années 60 . Il évoque le livre de 1997 de Jeannine Benyus "Bio-mimicry: innovation inspired by Nature » en indiquant qu'il faisait écho à ce que disait déjà en 1516 le grand Leonardo da Vinci : "prends tes leçons dans la nature, c'est là qu'est notre futur ». Selon Gilles Boeuf "on n'aurait jamais inventé un avion sans les chiroptères et les oiseaux; ce qui est fabuleux, c'est de voir que nos avions volent depuis 120 ans alors que nos oiseaux volent depuis 150 millions d'années! Quand on prend l'avion, depuis une quinzaine d'années, on constate que le bout des ailes est relevé ; des ingénieurs américains ont observé il y a une trentaine d'années les vols des grands rapaces qui relevaient le bout des ailes, les rémiges, dans les courants ascendants le long des falaises pour gagner de l'énergie et aller plus vite. On a ainsi inventé les winglets; or à l'époque, il n'y avait aucun travail de physique ou de mécanique qui accompagnait cela, on s'en est rendu compte une fois qu'on l'a fait et qu'on les a mises sur un avion car cela améliorait les performances de l'avion en faisant gagner entre 6 et $8 \%$ de carburant ». La bio-inspiration est une approche qui consiste à étudier la nature, à la scruter sous toutes ses formes : animaux, plantes, champignons, microorganismes (protistes et bactéries), écosystèmes au sens le plus large et à en s'en inspirer, pour des développements technologiques : matériaux et procédés novateurs au service de l'humanité, des productions moins polluantes, moins consommatrices d'énergie, recyclables, plus sûres, de meilleure qualité et à moindre coût. "Alors si tout cela ne fait pas rêver un chef d'entreprise!". Jeannine Benyus définissait à l'époque la bio-inspiration comme une démarche d'innovation faisant appel au transfert et à l'adaptation des principes et stratégies élaborés par des organismes vivants afin de rendre les sociétés humaines mieux compatibles avec la biosphère. "Vous voyez que le biomimétisme nous amène à un développement durable. Donc vous imaginez la puissance du système! La Vie s'est adaptée et pour ce faire, elle a compris depuis longtemps qu'il fallait changer! Et pourquoi nous, on ne s'adapte pas? Parce qu'on ne change pas. Or il faut changer! ». Gilles Boeuf indique qu'il vient d'écrire un ouvrage depuis la crise de la Covid : "Notre ennemi n'est pas le virus mais nous-mêmes " où il fait référence à " quelque chose qui n'aurait pas dî se passer et qui s'est tout même passé ", ainsi que le chapitre d'un livre intitulé " $L a$ Covid 19 : un électrochoc collectif salutaire ». Gilles Boeuf exprime ses craintes de voir un "monde d'après" ressemblant beaucoup au "monde d'avant": "Quand je vois aujourd'hui comment se profile ce que sera le post-Covid, je suis très inquiet. Quand va-t-on revenir au nombre d'avions qu'on avait avant? Le 20 mars 2020 il y a eu dans le ciel de la Terre plus de cent mille vols. Je suis désolé, il ne faut surtout pas revenir à cent mille vols. Je ne suis pas contre les avions mais simplement : où est la mesure? Et une atteinte par ce petit virus de quinze gènes (je vous rappelle que tous ici nous en possédons 29 000) comment cela se fait-il?». Gilles Boeuf regrette que la moitié des sols ait été tuée: "On ne nourrira jamais 8 milliards d'humains avec des sols morts. Il faut ramener de la manière organique dans les sols, ramener de la diversité biologique, fabriquer des matériaux, produire de l'énergie, nous soigner, stocker l'information et gérer nos entreprises. Voilà le défi du biomimétisme et de la bio-inspiration». Il énumère les "plaies écologiques " actuelles : changement climatique, productivisme agricole, pénurie d'eau, surpêche, déforestation, effondrement $\mathrm{du}$ vivant et pollution. Gilles Boeuf donne un exemple emblématique de source d'inspiration : une petite libellule odonate qui pèse 1,5 grammes, présente depuis 345 millions d'années, capable d'effectuer 20 millions de battements d'ailes dans sa vie, soit pendant quelques semaines à peine. Elle peut résister à d'importantes forces physiques et vole à $90 \mathrm{~km} / \mathrm{h}$ avec 2 Watts et voit 300 images par seconde à $360^{\circ}$. Elle dispose de capteurs de vol qui font rêver tous les pilotes et peut encaisser en accélération $30 \mathrm{G}$ ! «Alors au lieu de mettre des saletés dans les mares et faire des parkings par-dessus, allons voir comment fait une libellule! Il faut tuer notre arrogance et notre cupidité. Je pense que la bio-inspiration est le remède à tout cela : aller chercher dans le vivant des solutions à nos problèmes. ". Il évoque également le Shinkansen, train japonais le plus rapide du monde, qui a été complètement bio-inspiré par la tête et le bec du martin-pêcheur. «Vous avez vu comment un martin-pêcheur vole et entre dans l'eau, sans aucun frisson de la surface de l'eau. S'il y a un tremblement de la surface : il ne mange pas car le poisson a fui. Malheureusement ce train, construit de cette façon faisait beaucoup de bruit donc on a fait une seconde bio-inspiration. Pour le revêtement du train on s'est inspiré des interconnexions entre les plumes de hibou. Parce que le hibou, s'il fait du bruit, c'est pareil : il ne mange pas car cela fait longtemps que la musaraigne a fui. Avec ces deux bio-inspirations, on a le train le plus rapide de la planète et qui ne fait pas de bruit! " Gilles Boeuf cite enfin des biomatériaux qu'il considère comme extraordinaires : la chitine et la sclérotine qui sont "bien meilleurs que le carbonate de calcium de nos os » ainsi que des adhésifs : les pattes des geckos qui grimpent sur une vitre et les colles biologiques de byssus de moules. Il achève sa conférence en informant de la création du CEEBIOS (centre d'études et d'expertise sur le biomimétisme et la bio-inspiration).

A l'issue de ces deux conférences, la médaille de l'Académie vétérinaire a été remise par le Président aux deux conférenciers.

\section{BIBLIOGRAPHIE}

- Benyus J. Biomimicry: innovation inspired by Nature. Harper Perennial, 2002

- Boeuf G. Biomimétisme et bio-inspiration. Victoires Editions, 2014

- Orsenna E \& Saint Aubin I de. Géopolitique du moustique. Fayard, 2017

- Orsenna E. Cochons, voyage aux pays du Vivant. Fayard-Stock, 2020 